Theoretical and Mathematical Physics, 168(1): 857-857 (2011)

\title{
NONLINEAR PHYSICS: THEORY AND EXPERIMENT VI
}

In this issue, we complete the publication of works from the international workshop "Nonlinear Physics: Theory and Experiment VI."

The following reports were published in Vol. 167, No. 3:

M. Beccaria, G. Macorini, and A. Tirziu - Semiclassical short strings in $\operatorname{AdS}_{5} \times S^{5}$

L. V. Bogdanov - Interpolating differential reductions of multidimensional integrable hierarchies

F. Calogero - A new goldfish model

V. G. Dubrovsky, A. V. Topovsky, and M. Yu. Basalaev - New exact solutions of two-dimensional integrable equations using the $\bar{\partial}$-dressing method

V. S. Gerdjikov, G. G. Grahovski, A. V. Mikhailov, and T. I. Valchev - Rational bundles and recursion operators for integrable equations on A.III-type symmetric spaces

I. T. Habibullin and E. V. Gudkova - An algebraic method for classifying S-integrable discrete models

L. A. Kalyakin, O. A. Sultanov, and M. A. Shamsutdinov - Asymptotic analysis of a model of nuclear magnetic autoresonance

A. V. Kiselev and J. W. van de Leur - Variational Lie algebroids and homological evolutionary vector fields

B. G. Konopelchenko and G. Ortenzi - Birkhoff strata of the Grassmannian $\mathrm{Gr}^{(2)}$ : Algebraic curves

A. Kundu - Integrable twofold hierarchy of perturbed equations and application to optical soliton dynamics

G. Macorini and M. Beccaria - Reciprocity of higher conserved charges in the $\mathfrak{s l}(2)$ sector of the $\mathcal{N}=4$ supersymmetric Yang-Mills theory

L. Martina - Noncommutative mechanics and exotic Galilean symmetry

C. Scimiterna and D. Levi - Integrability of differential-difference equations with discrete kinks

V. A. Verbus, L. Martina, and A. P. Protogenov - Chain of interacting $S U(2)_{4}$ anyons and quantum $S U(2)_{k} \times \overline{S U(2)_{k}}$ doubles

Translated from Teoreticheskaya i Matematicheskaya Fizika, Vol. 168, No. 1, p. 3, July, 2011. 